\title{
Histologic Evaluation of Periodontal Implants in a Biologically "Closed" Model
}

\author{
Fred L. Bye, ${ }^{\star}$ Michael E. Krause, $†$ Joseph A. Regeził and \\ Raul G. Caffesse§
}

Accepted for publication 29 April 1986

\begin{abstract}
TO STUDY THE BIOLOGIC RESPONSE, three commercial calcium phosphate implant materials (Calcitite", Periograf", Synthograft") were implanted in cuspid root "windows" in four beagle dogs. No implant material was placed in the fourth cuspid window which served as a control. Following mucoperiosteal flap elevation, windows were chiseled in bone to the root surface which allowed implant particles to contact bone, fibrous connective tissue, cementum and dentin without exposure to the oral environment. Animals were sacrificed after 1, 3, 5 and 6 months.

Histologically, all implant materials were well tolerated. At 1 month, implant particles were surrounded by fibrous tissue. Fibrous tissues filled the control defect. At 3 months, implant sites exhibited partial bony repair with connective tissue surrounding implant particles. A ring of osteoid surrounded Synthograft particles. Control defect repair was complete. At 5 and 6 months, implant sites exhibited advanced, though incomplete, bony repair. New bone encompassed the Synthograft particles. It was concluded: 1) Control sites healed most rapidly. 2) Calcitite and Periograft were well tolerated space occupiers. 3) Synthograft was a nidus for bone deposition.
\end{abstract}

Alloplastic periodontal grafting materials have been used to stimulate the regeneration of supporting bone lost from periodontal disease. Ideally, this restoration of lost alveolar bone should occur by either direct replacement or induction. Accordingly, the therapeutic value of any synthetic calcium phosphate implant material must depend upon some means of inducing new bone formation.

Calcium phosphate implants have not been found to induce new bone formation in the traditional sense. ${ }^{1}$ However, they have displayed bone growth "guidance" properties by causing bone to grow into areas it would otherwise not occupy. ${ }^{3}$ This property has been termed "osteoconductivity." In a series of investigations, the repair of nonperiodontal surgically created defects in dogs was found to be more rapid when filled with resorbable tricalcium phosphate implants in comparison to unfilled control defects. ${ }^{2}$ However, investigators who surgically created periodontal defects in dogs found

\footnotetext{
* Assistant Professor, Department of Periodontics, The University of Michigan School of Dentistry, Ann Arbor, MI 48109.

+ Private Practice, 1120 W. Huron, Ann Arbor, MI.

¥ Professor of Dentistry, Department of Pathology, The University of Michigan School of Dentistry.

$\S$ Professor and Chairman, Department of Periodontics, The University of Michigan School of Dentistry.
}

a more rapid and complete filling of control sites as compared to porous nonresorbable hydroxylapatite ${ }^{4}$ or porous resorbable tricalcium phosphate implants. ${ }^{5.6}$ In contrast to the above, similar rates of healing were found in a study comparing unfilled surgically created periodontal defects with defects containing dense nonresorbable hydroxylapatite implants ${ }^{7}$

The histologic features associated with calcium phosphate implantation have included a negligible inflammatory or foreign body response. ${ }^{8}$ New bone deposition has been found to occur either directly upon the calcium phosphate implant surface ${ }^{8.9}$ or adjacent to an intervening fibrous tissue capsule surrounding the implant. $^{6}$ Varying rates and degrees of resorption of different calcium phosphate implants have also been described depending upon their composition and/or density. ${ }^{3}$

The purposes of this study were to use a periodontal model in dogs where communication with the oral environment was prevented, and to histologically evaluate the biologic response to three commercially available calcium phosphate implant materials compared to unfilled control sites. A biologically "closed" system essentially eliminated any potentially adverse influence of bacterially induced marginal inflammation during the healing process and thereby allowed comparisons 
of the different implant materials under similarly ideal healing conditions.

\section{MATERIALS AND METHODS}

Four 5 to 7-year-old female beagle dogs were used in this study. Each exhibited generalized moderate periodontitis with 4 to $5 \mathrm{~mm}$ pockets and bone loss 3 to 4 $\mathrm{mm}$ apical to the cementoenamel junctions (CEJs) as determined radiographically. Hygienic phase procedures consisted of a single scaling and root planing session, and thereafter toothbrushing was done 3 times weekly throughout the duration of the investigation. The animals were fed a hard diet of Purina Dog Chow and water ad libitum.

One month following scaling and root planing, surgery was performed on the facial aspects of all maxillary and mandibular cuspid teeth. Pocket depths for the cuspid teeth did not exceed $3 \mathrm{~mm}$ and bleeding was not elicited upon gentle probing. The animals were anesthetized with acepromazine and sodium pentobarbital. Following local infiltration anesthesia (Xylocaine ${ }^{\text {tR }}$ with $1 / 50,000$ epinephrine), a vertical releasing incision was made followed by mucoperiosteal flap elevation to expose approximately $1 \mathrm{~cm}$ of facial supporting alveolar bone, apicoincisally. A rectangular "window" $(5 \times$ $5 \mathrm{~mm}$ ) was next chiseled through the alveolar bone to the root surface over the midsection of the cuspid root (Fig. 1). The method of treatment was then randomly assigned as either Calcitite, ${ }^{*}$ Periograf, $\dagger$ Synthograft $\ddagger$ or control. Each of the three implant materials was placed into a separate "root window" in each dog. The flap was then reapproximated near its preoperative level and sutured to completely cover the surgical site with extreme care being taken not to displace the implant particles. Firm finger pressure with moist saline gauze was maintained for 5 minutes for additional implant particle stabilization. The implant materials, thereby, simultaneously contacted bone, fibrous connective tissue, cementum and dentin (in areas of inadvertent cementum removal during window preparation). The fourth cuspid window in each animal served as a control with no graft material placed into this surgical defect. These windows provided the opportunity to evaluate the biologic response to the calcium phosphate implant materials in an essentially inflammation-free periodontal environment isolated from the oral environment.

The animals were then maintained on a soft diet with toothbrushing every other day until the sutures were removed 1 week postoperatively. Antibiotic therapy was not given postoperatively. After the 1st week, the animals were returned to their preoperative diet and oral hygiene regime. The animals were sacrificed after

\footnotetext{
* Calcitek, Inc, San Diego, CA.

+ Cook-Waite Laboratories, Inc., New York, NY.

$\ddagger$ Miter, Inc, Indianapolis, IN.
}

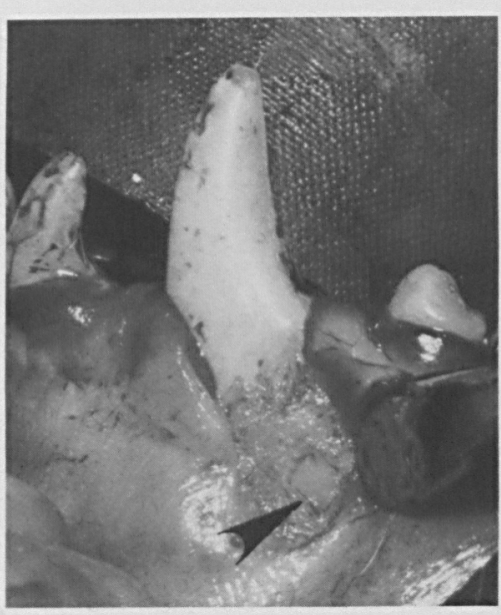

Figure 1. Clinical photograph of prepared cuspid "window" (arrow) in beagle dog. Note "window" is surrounded by alveolar bone.

1-, 3-, 5- and 6-month postoperative intervals. Tissue was formalin-fixed, decalcified in $20 \%$ formic acid and embedded in paraffin. Sections were stained with hematoxylin and eosin and evaluated microscopically. A cross-sectional orientation of the specimens was used during all histological evaluations.

\section{RESULTS}

Clinically, no adverse reactions to the implant materials were apparent during the six-month postoperative period. Surgical wounds healed without complication. There was no swelling or redness associated with the implant sites.

Histologically, all implant materials could be identified in the surgical defects and were distinguishable from each other only by their characteristic particle shapes. Calcitite particles were irregularly shaped with rounded, smooth margins (Fig. 2A). Periograf particles were identifiable as being more angular and less regularly shaped (Fig. 5), while Synthograft particles were spherical in shape (Fig. 6).

One-Month Results. The dense connective tissue stroma surrounding the Calcitite particles (Fig. 2A) was representative of the 1-month findings for all grafted materials. In Figure 2A the bony margin of the surgically created defect was in close proximity to one of the implant particles, with the periodontal membrane found to be artifactually separated from the underlying root surface. The osseous defect margins exhibited osteoblastic hyperplasia and osteoid formation (Fig. 2B). Osteoclasts were also observed in the remodeling process.

At 1 month, the unfilled control defects were completely filled with fibrous connective tissue. Similar to the experimental sites, osteoblasts and osteoid were observed at the defect margins.

Three-Month Results. After 3 months, both Calcitite and Periograf particles continued to be closely sur- 

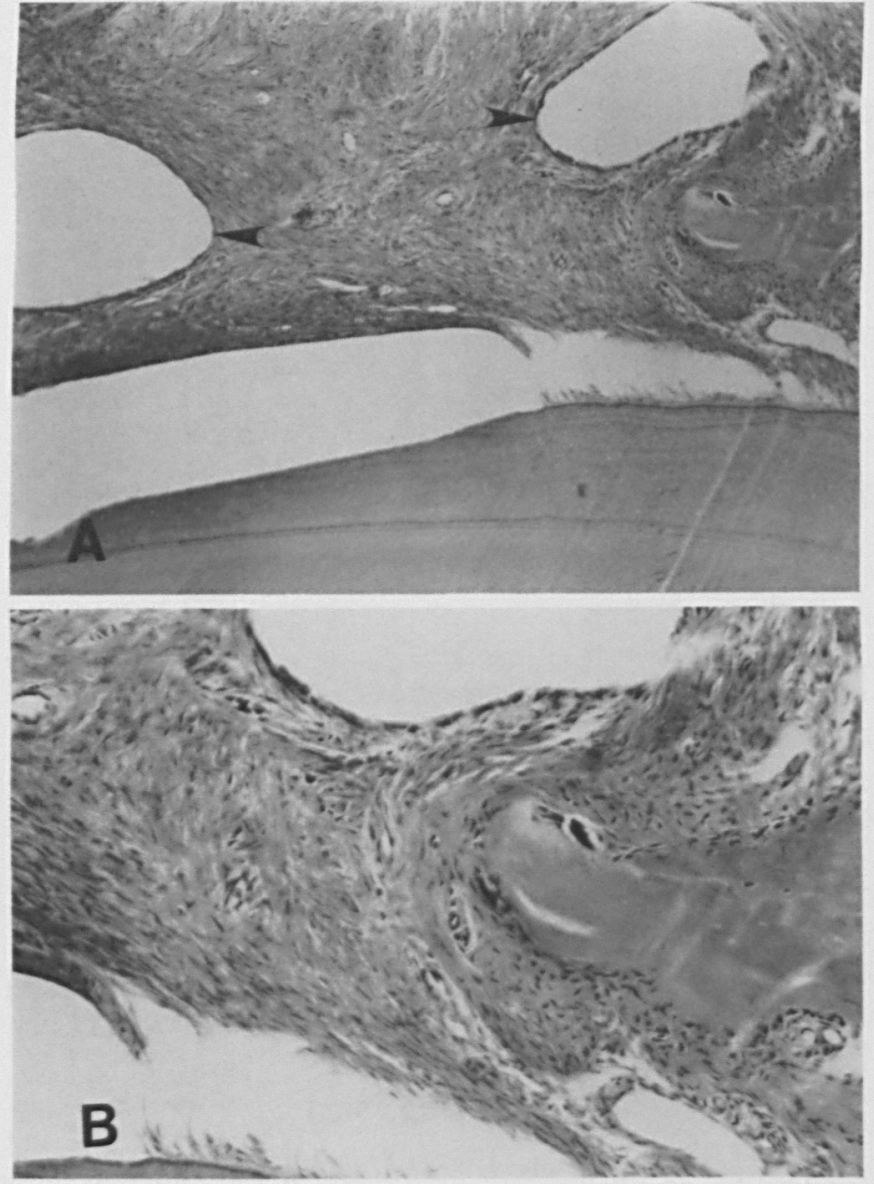

Figure 2. A. Two-rounded irregularly shaped Calcitite particles (arrows) surrounded at I month by a dense connective tissue stroma. The osseous defect margin is in close proximity to one of the implant particles (right side) with the periodontal membrane artifactually separated from the underlying root surface (magnification $\times 100$ ). $\mathbf{B}$. Higher magnification of the osseous defect margin showing osteoblast hyperplasia and osteoid formation. Note implant particle above (mag nification $\times 250$ ).

rounded by fibrous connective tissue. Partial bony repair at the defect margins was observed in the form of osteoblasts with adjacent osteoid formation. There was also evidence of new cementum in all defects.

After 3 months, the Synthograft experimental sites presented an additional finding. The Synthograft particles were surrounded by a ring of eosinophilic material suggestive of osteoid (Fig. 3). Cells with dark-staining nuclei consistent with osteoblasts were observed in close approximation to the eosinophilic material.

Bony repair was essentially complete after 3 months in the control sites where no calcium phosphate particles had been placed (Fig. 4). The presence of increased numbers of osteoblasts and the marked cellularity of the periodontal membrane was indicative of the active repair which occurred within the control defects.

Five- and 6-Month Results. At 5 and 6 months, advanced, though incomplete, bony repair was evident at all experimental sites. Both Calcitite and Periograf (Fig. 5) implant particles continued to be surrounded by a fibrous connective tissue stroma.

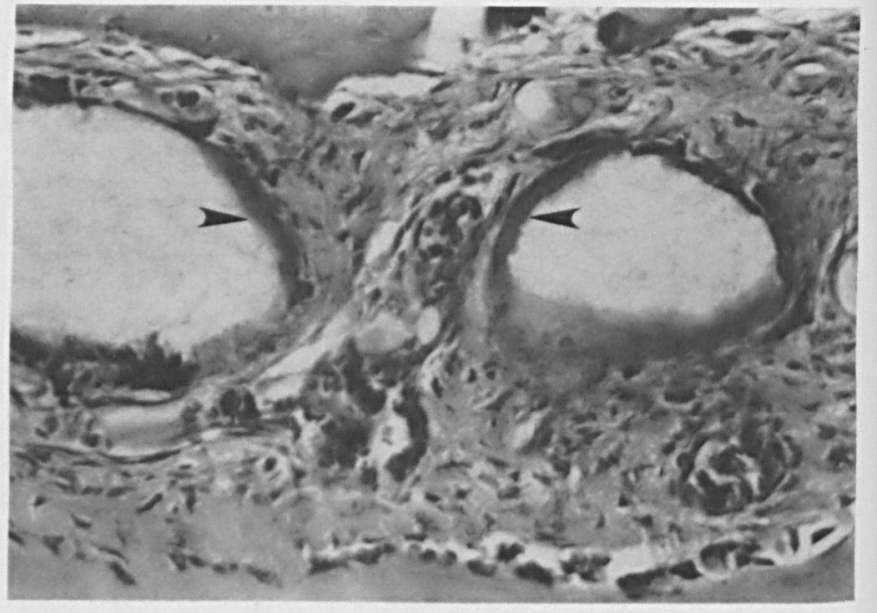

Figure 3. Synthograft implant site at 3 months showing two spherical implant particles being surrounded by an eosinophilic material (arrow's) suggestive of osteoid (magnification $\times 400$ ).

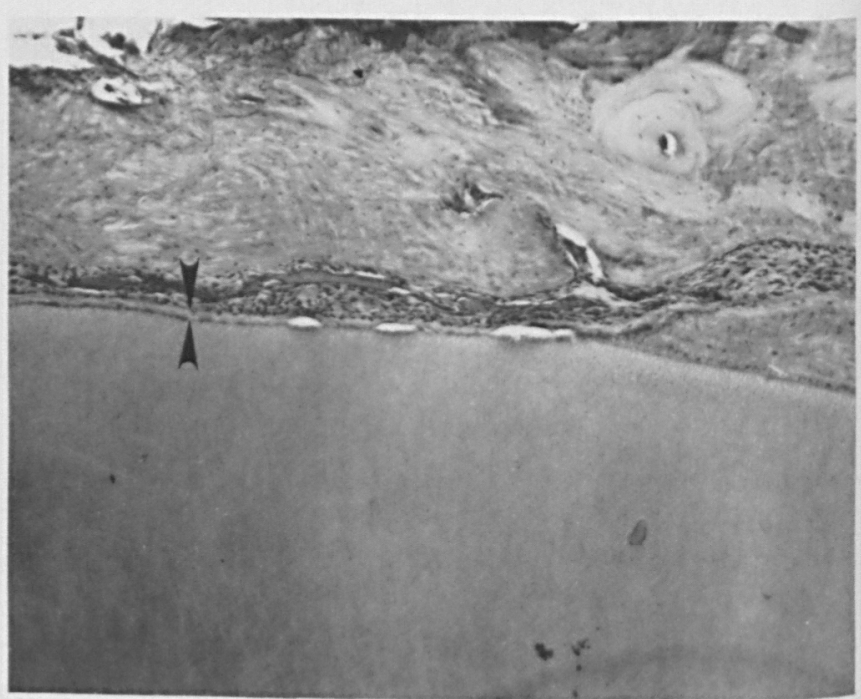

Figure 4. Control defect at 3 months showing repair. Note thinned cementum following surgical preparation (arrow's) (magnification $\times$ 100)

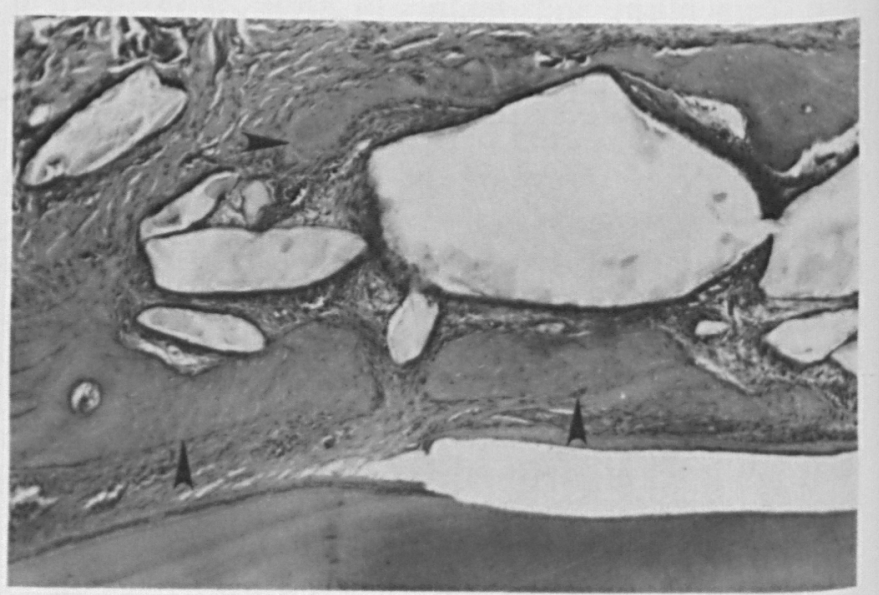

Figure 5. Site of surgical defect at 6 months showing new islands of bone (arrow's) achieved with angular Periograf particles. Note tooth at bottom of photomicrograph adjacent to separation artifact (magnification $\times 250$ ) 
After 5 and 6 months, the Synthograft sites still presented a unique appearance (Fig. 6) compared to areas of Periograf and Calcitite implantation. New bone, as evidenced by osteocytes and osteoid, encompassed the spherical Synthograft particles, which in some areas became contiguous with the regenerated alveolar bone. Synthograft particles could be seen surrounded by hard tissue (Fig. 7) considered to be new cementum due to its continuity with the root surface.

The 5- and 6-month control sites exhibited an appearance similar to that found after 3 months when bony repair was considered complete.

\section{DISCUSSION}

The rate of surgical repair of control defects as compared to calcium phosphate implanted defects has been found to vary depending upon the type of implant material ${ }^{5.7}$ and location of the defect site. ${ }^{2.5}$ In a study of nonresorbable hydroxylapatite implant material

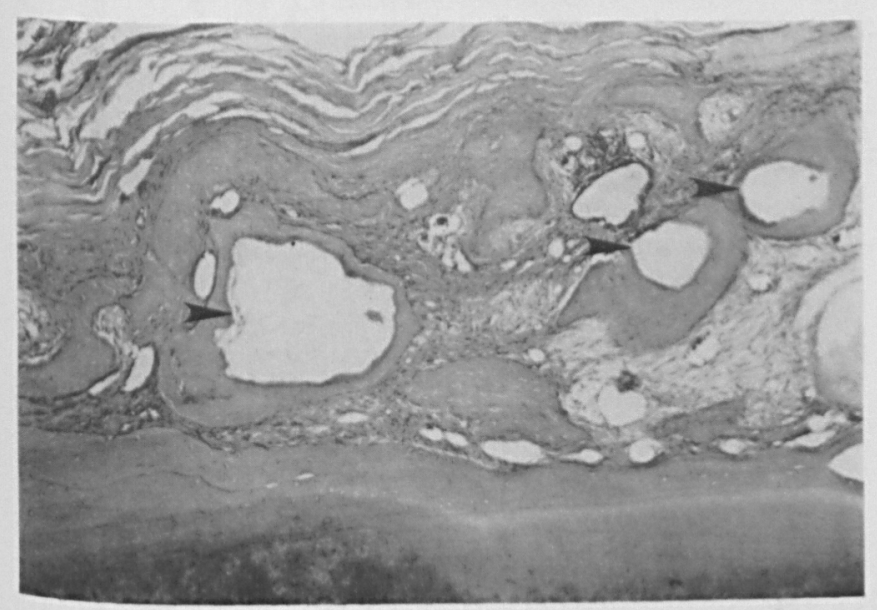

Figure 6. Synthograft implant site at 6 months showing spherical implant particles (arrows). Implant material is surrounded by bone and is continuous with alveolar bone (left side). Dentin and cementum of tooth are noted below (magnification $\times 100$ ).

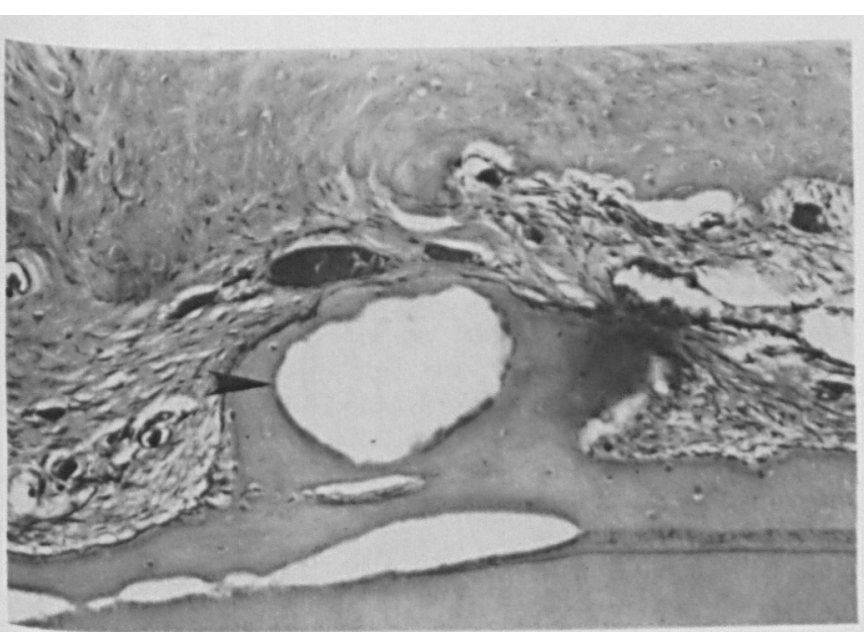

Figure 7. Sinthograft implant site at 6 months showing implant particle (arrow) incorporated into repaired cementum of $t(x) t h$ below. (magnificarion $\times 250)$. placed into surgically created periodontal defects in dogs, the empty control sites demonstrated complete healing with new bone after 16 weeks, whereas the implanted sites still contained large amounts of proliferating fibrovascular tissue. ${ }^{4}$ Similar results were obtained in the present study with bony repair of the unfilled control defects being essentially complete after 3 months. In contrast, healing of the calcium phosphate implant sites was relatively incomplete through the 6month duration of the investigation. In this closed, virtually inflammation-free, dog-periodontal-defect model, the three commercially available calcium phosphate implants evaluated were well-tolerated clinically and histologically, but did not hasten the healing process.

The bone guiding or "osteoconductive" properties of the calcium phosphate implants have been discussed by several investigators. ${ }^{2.5 .10}$ Histologic evidence of bone deposition either in direct contact with the calcium phosphate implant ${ }^{4.8 .10}$ or within the adjacent connective tissue ${ }^{5.6}$ has frequently been suggested to be due, at least in part, to the presence of the implant material. However, direct bone apposition against the implant surface may simply reflect the inert, noninflammatory and highly biocompatible nature of calcium phosphate implants $^{12.14}$ rather than any inherent bone inducing capability of the implant. Other investigators have regarded the fibrous connective tissue frequently observed surrounding the implants to be more of a fibrous scar ${ }^{11}$ or capsule ${ }^{22.13}$ with the implant acting as a biocompatible "filler." In this study, because the nonresorbable hydroxylapatite implants (Calcitite and Periograf) were always surrounded by fibrous connective tissue and did not appear to be directly influencing bone deposition, they could be also regarded as biocompatible "filler."

Other investigators found that when resorbable tricalcium phosphate implants were used to fill intrabony defects in dogs, after 6 weeks, an eosinophilic "collagenlike" material was found surrounding islands of what the authors interpreted as increased collagen formation. ${ }^{5}$ While these ceramic-filled defects healed slower than the unfilled control defects, the investigators felt the implants had initiated new bone formation. After 3 months, the "resorbable" tricalcium phosphate implant particles used in this study (Synthograft) were found to be associated with a ring of eosinophilic material consistent with osteoid. The unique appearance of the tricalcium phosphate implants was persistent after 6 months with the presence of new bone, as evidenced by osteocytes and osteoid, encompassing the graft particles. Whether the observed presence of new bone directly approximating the tricalcium phosphate particles represented bone guidance or was simply the result of normal osseous repair adjacent to a highly biocompatible material undergoing resorption cannot be determined from this study. Also, the potential for the induction of ankylosis at the implant tooth interface 
could not be determined. ${ }^{3}$ Further investigation of this interaction between hard tissues and "resorbable" tricalcium phosphate appears warranted.

It may be concluded, within the limits of this study, that:

1. Control sites healed more rapidly than experimental sites.

2. The hydroxylapatite implants (Calcitite and Periograf) caused a negligible inflammatory response and periparticle fibrosis. They may be regarded as well-tolerated space occupiers.

3. The tricalcium phosphate implant (Synthograft) caused a negligible inflammatory response and appeared to serve as a nidus for bone and cementum deposition.

\section{REFERENCES}

1. Urist, M. R., and Stiates, B. S.: Bone morphogenetic protein. $J$ Dent Res 50: 160, 1973.

2. Ferrara, J. W.: Experimental evaluation of ceramic calcium phosphate as a substitute for bone grafts. Plast Reconstr Surg 63: 634, 1979.

3. Meffert, R. M., Thomas, J. R., Hamilton, K. M., and Brownstein, C. N.: Hydroxylapatite as an alloplastic graft in the treatment of human periodontal osseous defects. J Periodontol 56: 63, 1985.

4. Nery, E. B., Lynch, K. L., Hirthe, W. M., and Mueller, K. H.: Bioceramic implants in surgically produced infrabony defects. $J$ Periodontol 46: 328, 1975.

5. Levin, M. P., Getter, L., and Cutright, D. E.: A comparison of iliac marrow and biodegradable ceramic in periodontal defects. $J$ Biomed Mater Res 9: 183, 1975.
6. Levin, M. P., Getter, L., Adrian, J., and Cutright, D. E.: Healing of periodontal defects with ceramic implants. J Clin Periodontol 1: 197, 1974.

7. Boyne, P. J., and Shapton, B. A.: The response of surgical periodontal defects to implantation with a hydroxylapatite ceramic. Trans 4th Annu Meet Soc Biomater 10th Int Biomater Symp p. 115, 1978.

8. Jarcho, M., Kay, J. F., Gumaer, K. I., et al.: Tissue cellular and subcellular events at a bone-ceramic hydroxylapatite interface. $J$ Bioeng 1: 79, 1977.

9. Kato, K., Aoki, H., Tadata, T., and Ogiso, M.: Biocompatibility of apatite ceramics in mandibles. Biomater Med Devices Artif Organs 7: 291, 1979.

10. Kent, J. N., Quinn, J. H., Zide, M. F., et al.: Correction of alveolar ridge deficiencies with nonresorbable hydroxylapatite. $J \mathrm{Am}$ Dent Assoc 105: 993, 1982.

11. Beirne, O. R., and Greenspan, J. S.: Histologic evaluation of tissue response to hydroxylapatite implanted on human mandibles. $J$ Dent Res 64: 1152, 1985.

12. Moskow, B. S., and Lubarr, A.: Histologic assessment of human periodontal defect after Durapatite ceramic implant. Report of a case. J Periodontol 54: 455, 1983.

13. Froum, S. J., Kushner, L., Scopp, I. W., and Stahl, S. S.: Human clinical and histologic responses to Durapatite implants in intraosseous lesions. J Periodontol 53: 719, 1982.

14. Cohen, D. W., Yukna, R. A., Scopp, I. W., et al.: A symposium on a new bone-grafting implant material for the treatment of periodontal disease. Compend Cont Educ Dent (Spec. suppl.) Jan.-Feb., 1982.

Send reprint requests to: Dr. Fred L. Bye, Department of Periodontics, University of Michigan, School of Dentistry, Ann Arbor, MI 48109 .

\section{Abstracts}

\section{A Comparative Study of the Desensitizing Effects of DENTIFRICES CONTAINING SODIUM MONOFLUOROPHOSPHATE AND FORMALIN}

Perminder, K., Shahani, D. R., and Antia, F. E. $J$ Indian Dent Assoc 57: 453, November, 1985

A double-blind clinical study comparing the desensitizing effects of dentifrices containing $0.76 \%$ sodium monofluorophosphate (MFP) and $1.3 \%$ formalin was conducted on three groups of 30 patients each. One group served as a control and used a placebo. Each group was instructed to use the assigned dentifrice with a toothbrush twice daily for 5 minutes for a 6-week period. Using a Telethermometer, sensitivity to hot and cold was tested by increasing or decreasing the temperature to the point where pain was felt. These values were compared to baseline measurements. Results concluded that no differences were found between the dentifrices for hot stimuli; however, the MFP dentifrice was more effective than the formalin dentifrice as far as cold response was concerned. College of Dental Surgery, Manipal 576 119. Karnataka. India.

Dr. Craig Goodman

\section{ASSOCIATION OF LEUKOPLAKIA WITH ABO BLOOD GROUPS IN} SOUTHERN ORISSA

Vaish, R. P., Jena, D. C., Panigrahi, R. K., and Panda, R. P. $J$ Indian Dent Assoc 57: 459, November, 1985

Leukoplakia was diagnosed in 383 patients out of 10,000 people, screened above the age of 14 , who were examined irrespective of complaints. ABO blood grouping was performed using high titre anti$A$ and anti-B group sera by the slide method in the 383 cases diagnosed. The normal control for blood group distribution was recorded from the records of healthy volunteers to the blood bank. Findings included a male predilection over females by a ratio of $3.4: 1$. The maximum number of cases $(32.7 \%$ ) of leukoplakia were detected among males between the ages of 40 to 49 years and among females between 50 to 59 years. The number of people belonging to blood group A with leukoplakia was significantly greater than that in the control group. In all other blood groups, no significant correlations to controls were reported. MKCG Medical College, Berhampur (Gm.), Orissa. India.
Dr. Craig S. Goodman 